(C) 2009 IEEE. Personal use of this material is permitted. Permission from IEEE must be obtained for all other uses, in any current or future media, including reprinting/republishing this material for advertising or promotional purposes, creating new collective works, for resale or redistribution to servers or lists, or reuse of any copyrighted component of this work in other works. 


\title{
Seamless Integration of Unidirectional Broadcast Links into QoS-constrained Broadband Wireless Mesh Access Networks
}

\author{
Mathias Kretschmer, Gheorghita Ghinea \\ Fraunhofer FOKUS, Germany \\ Brunel University London, United Kingdom \\ mathias.kretschmer@fokus.fraunhofer.de,george.ghinea@brunel.ac.uk
}

\begin{abstract}
Wireless Mesh Networks (WMNs) have matured in recent years. They allow two nodes to communicate via multiple hops when no direct connectivity exists. This allows community networks such as Freifunk [3] to provide affordable Internet access to their communities by sharing a few gateway nodes. Increasing demand for multi-media content poses a challenge for the typical WMN routing protocols such a Optimised Link State Routing (OLSR) which lack Quality of Service (QoS) support. Although, numerous solutions have been proposed to introduce QoS awareness such as [4], those WMNs fail to meet commercial operator requirements in term of reliability and QoS guarantees. In this paper we propose the seamless integration of Broadcast technologies such as Digital Video Broadcast (DVB) to introduce support for high-bandwidth multi-media services.
\end{abstract}

\section{Motivation}

The visibility and success of WMN deployments has raised interest among commercial operators in this technology. Compared to traditional operator access networks WMNs have the potential to offer easier deployment, flexible self-configuration at lower costs. A WMN-type architecture considered as an alternative for an operator access network must meet similar requirements such as guaranteed QoS and high availability. Such a Carrier-grade Wireless Mesh Access Network (CG-WMAN) can assume more reliable and higher performing hardware yielding more stable links.

Thus, CG-WMANs can be compared to a typical operator access network with two major limiting factors: the severe capacity constraints due to scarce wireless spectrum resources and temporarily varying link conditions. An operator access network needs to support triple-play services (web, video, voice) which puts an emphasis on QoS guarantees and efficient resource utilization in a capacityconstrained network. Resource utilization can be optimized by using the most suitable technology for a given payload. For example, the delivery of live multimedia content is facilitated by only one transmitter, hence the overhead of Media Access Control (MAC) protocols can be avoided. Unidirectional technologies such as Digital Video Broadcast -Terrestrial (DVB-T) offer a very efficient distribution medium for 1-to-N traffic and usually span across multiple mesh hops. Typically, WMNs rely on the bi-directionality of links and can therefore not utilize Unidirectional Links (UDLs). In this paper we propose a link layer approach to seamlessly integrate UDLs into CG-WMANs so that higher layer protocols can transparently utilize them when beneficial for a given payload or receiver distribution.

\section{Seamless Integration of Unidirectional Links into a CG-WMAN}

UDLs are a result of technology specific link layer characteristics; hence, we propose to address them at the link layer where they occur. This allows higher layer CG-WMAN protocols to utilize UDLs as regular links. Combined with Multi Protocol Label Switching (MPLS)[6] we provide support for QoS-constraint routing, unlike approaches such as Bidrectional Routing Abstraction (BRA)[5] or Unidirectional Link Routing (UDLR)[2] which only address 'best effort' connectivity.

\subsection{Heterogeneous Technologies}

Our approach integrates heterogeneous technologies via MPLS on the data plane. The control plane relies on IEEE 802.21 to support local as well as remote management of heterogeneous mesh nodes and their radio interfaces. We will identify additional 802.21 features where required, and discuss them in the 802.21 mesh ad-hoc group. Similarly, we will continue our contribution to the 802.21 b study group which addresses the integration of DVB technologies.

\footnotetext{
The work described here was carried out within the EU-ICT FP7 project CARMEN "Carrier Grade Wireless Mesh networks, contract number: 214994"
} 


\subsection{Topology Discovery and Self- Management}

Topology discovery is initiated and controlled by gateway nodes which try to discover all nodes in their vicinity as well as all possible physical links between nodes. They also form areas to establish a two-layer hierarchy keeping topology change information local. Existing approaches such as using inclusive cycles [1] to detect UDLs will be evaluated for their applicability. Out of the set of physical links, the self-management component computes and maintains a subset of optimal logical links to be used for mesh traffic. Logical links are described as a unidirectional resource to aid traffic management which also works unidirectionally.

\subsection{Link Monitoring}

Link monitoring in our work is a crucial component of a CG-WMAN ensuring that wireless links operate within their expected parameters. Underperforming links are reported to the gateway which computes and triggers reconfigurations. The monitoring component also passively monitors the node neighborhood to detect interfering nodes, external noise sources or new mesh nodes joining the network. The following questions need to be addressed to ensure equivalent operation over UDLs:

- Use of passive monitoring instead of ACKs ?

- Signaling of broken links to gateway nodes ?

- How to trigger MPLS Fast Reroute (FRR) upstream?

\subsection{QoS Path Computation and Forwarding}

At each gateway, our work uses a fully centralized and stateful QoS-constrained path computation module employing the Dijkstra algorithm which natively considers UDLs if present in the link state table. This table is derived from the set logical links available for mesh traffic. Protocols such as Open Shortest Path First (OSPF) or Intermediate system to intermediate system (IS-IS) do not store UDLs. Path computation can be performed for unicast paths as well as for 1-to-N multicast trees. The resulting forwarding state is then pushed into the intermediate mesh nodes along the path from the source towards the destination(s).

For MPLS path setup or tear down a protocol such as Resource ReSerVation Protocol-Traffic Engineering (RSVPTE) is required to signal a path between arbitrary mesh nodes. Since only the gateways maintain topology information, source routing is used to direct the RSVP-TE messages from the source to the sink and vice versa. To achieve this, RSVP-TE needs to be extended by a source routed return path object. To support fast failovers, MPLS FRR will be considered. Careful considerations are required in a CG-WMAN due to the trade-off between having fall-back paths vs. extra capacity allocation.

\section{Conclusion and Future Work}

The data link layer seems most suitable for the seamless integration of UDLs into a CG-WMAN. Similar to the 802.11 s extension, this requires some network layer functionality to be pushed into the data link layer. The advantage of that is that rather more complicated network layer protocols such as Mobile IP do not need to be modified. Future work will focus on the efficient utilization of DVB overlay cells in a CG-WMAN. Instead of relying on the same simple metric used for mesh links, we will investigate modern decision making algorithms to balance between the requirements of the payload, the topological distribution of the receivers and the costs of overlay cell resources compared to mesh links.

\section{References}

[1] L. Bao and J. J. Garcia-Luna-Aceves. Link-state routing in networks with unidirectional links. In Proc. Eight International Conference on Computer Communications and Networks, pages 358-363, 1999.

[2] E. Duros, W. Dabbous, H. Izumiyama, N. Fujii, and Y. Zhang. A Link-Layer Tunneling Mechanism for Unidirectional Links. RFC 3077 (Proposed Standard), Mar. 2001.

[3] Freifunk Community. http://www.freifunk.net. Accessed 25-May-2009.

[4] V. Kone, S. Das, B. Y. Zhao, and H. Zheng. Quorum -quality of service routing in wireless mesh networks. In ICST Conference on Heterogeneous Networking for Quality, Reliability, Security and Robustness (QShine), 2007.

[5] V. Ramasubramanian and D. Mosse. Bra: A bidirectional routing abstraction for asymmetric mobile ad hoc networks. IEEE/ACM TON 16(1):116-129, 2008.

[6] E. Rosen, A. Viswanathan, and R. Callon. Multiprotocol Label Switching Architecture. RFC 3031 (Proposed Standard), Jan. 2001. 